\title{
Research on Visual Evaluation Method of Construction Quality Based on BIM
}

\author{
Yi Xiuhonga , Wang Yongli \\ Jilin Railway Technology College, Jilin, China, 132001 \\ a.24702296@qq.com
}

Keywords: BIM, Visual Evaluation, Construction Quality

\begin{abstract}
After the research, analysis and comparison of several architectural visualization technologies commonly used in the field of architectural design, the method for visualizing BIM design results is discussed, the practical value of visual outcome applications is presented, and the application value of BIM technology system in the field of construction engineering is enriched.
\end{abstract}

\section{Introduction}

With the rapid development of computer graphics technology and information technology, building information modeling (BIM) technology has become a popular technology in the current construction engineering industry. BIM is based on a three-dimensional collaborative design model. The design results carry a large amount of information and will bring new value experiences to the parties involved in all phases of construction. It is destined to become the mainstream technology in the field of construction engineering. The BIM 3D design model can better show the designer's intentions. The results can be directly visualized and applied, making the design review of the project more convenient and intuitive, avoiding the waste of repeated modeling of human resources and the delay of time. Therefore, building visualization It is an important application value recognized by BIM technology. Discuss several ways to visualize BIM design results, as well as their own characteristics, and discuss the main application fields of visual results.

\section{Quality Remote Inspection System for Construction Engineering}

With the help of the BIM Building Information Model, a new technology, the company will optimize and improve the quality supervision work, and carry out coordinated monitoring, feedback, rectification, and acceptance of remote projects on the cloud platform, urge all participating parties to implement the main responsibilities and enhance the modernization of supervision capabilities. Level, improve project quality and safety supervision effectiveness, guarantee the safety and quality of construction projects [1].

During the construction process of the construction project, various participating units will form a large number of materials such as capital construction documents, construction materials, supervision materials, and as-built drawings. The supervision of engineering quality supervision agencies includes the implementation of laws, regulations, and mandatory standards for project construction by relevant units. Spot checks and random surveys of the quality of the project entities involved in the safety and main functions of the engineering structures. The quality of major construction materials, architectural components and equipment, and equipment are checked and sampled. And other complex content. The project quality supervision process needs to do a lot of work, and forms a large number of electronic and paper supervision files. Supervision work and archives data governance have become important connotations for the modernization of supervision capabilities [2]. This article will carry on the combing research from the following aspects: First, the classification and combing of the traditional quality supervision work, according to the characteristics of the analysis of the feasibility of its information approach; Second, the structure of the information needed to provide inspection; We need to link and correspond components such as sub-projects and inspection lots that require quality supervision with necessary quality supervision documentation; and fourthly, a large number of tedious and repetitive manual comparisons in the 
traditional quality supervision process. , Through the built-in standard, enter the scene data to achieve automatic computer comparison, instead of manual operations.

The remote inspection of engineering quality mainly requires information and IT technologies: one is BIM (Building Information Model) technology; the other is component-based carrier and core integrated information linkage; and third, real-time monitoring of on-site status and virtual model synchronization; The fourth is multi-terminal, multi-user collaboration on an information model and the Internet cloud platform; Fifth, inspection standard presets and automatic verification. The system uses the Tianbao TX5 laser scanner and the Tianbao RTS873 measuring robot as ancillary equipment. The key technical highlights are: First, the loading and extraction of information in the BIM building information model; Second, the use of advanced high-precision positioning equipment and equipment combined with the BIM information model. , to improve the traditional way of operation; third is to use the Internet cloud platform integrated construction monitoring information system; fourth is to generate two-dimensional code for building components, component groups, participating parties can follow the two-dimensional code tracking, information entry, etc.; Visualization technology, VR technology; Six is dual-view synchronization technology [3].

\section{BIM architectural visualization application method}

Revit design software is currently one of the main BIM application software in China. As an example, there are two basic methods for visualizing software. One is to use "real" visual styles in 3D design views. This style displays the materials in Revit in real time. And textures to facilitate visualization during the design process. The other is to render the model to create a photorealistic image. Revit design software has its own technical elements such as materials, lighting, environmental scenery, and rendering. It uses the mental ray\&reg rendering engine to ensure high-quality rendering. The speed of rendering is affected by the number of model primitives, the level of detail, and the size of the rendered view area. The performance of the rendering is affected by the number of lights, the shape of the light source, the softness of shadows, indirect lighting, and the number of models controlled by the section boxes and light groups. With Revit's own rendering capabilities, any camera position and viewing angle can be set during the design process. The designer can directly obtain renderings and animation videos for reviewing the design.

With regard to the fineness and the size of the rendered view area, the performance of the rendering is affected by the number of lights, the shape of the light source, the softness of the shadows, indirect lighting, and the number of models controlled by the section boxes and light groups. With Revit's own rendering capabilities, any camera position and viewing angle can be set during the design process. The designer can directly obtain renderings and animation videos for reviewing the design [4].

In Autodesk's BIM software system, there is Navisworks, a lightweight visual management software that seamlessly interfaces with Revit. The Navis file retains the attribute information of Revit models and components, discards the association calculation between components, and the file size is streamlined and optimized. The software provides materials, lighting, basic environment, RPC objects and other settings, the speed and effect of rendering have also been improved, can produce high-quality renderings and animation, to meet the needs of visual inspection of the building. In addition, an environment based on real-time graphical interactive operation is provided to facilitate users to roam through the first-person view, forming an architectural visualization supporting application, helping designers to more efficiently check designs in real time. Therefore, it can be said that Autodesk's BIM software system is relatively complete, taking full account of the application requirements of architectural visualization in design.

BIM has good data interactivity and can export commonly used 3D data exchange format files, which can fully satisfy the data conversion between BIM models and other 3D authoring software. After importing professional three-dimensional animation production software, traditional visual production is performed to obtain high-quality images, animation videos, and generate virtual reality products for high-end display of buildings. Of course, in this way, the production is relatively 
cumbersome. In particular, the production of virtual reality requires more production steps and will also pay more time. The application of architectural visualization in the past has long been in the predicament of rendering high-quality images and taking a lot of time. This situation is closely related to the development level of computer hardware. In the past, graphic calculations were limited by the computing power of the graphics card. It is necessary to obtain more accurate image expression through CPU calculations. However, the graphic display is related to the computational performance of the GPU. As the GPU hardware performance improves, many software vendors are committed to using the GPU's computing power to achieve real-time display that is closer to the performance of the rendering, including architectural visualization software. Lumion is such an excellent real-time 3D visualization tool whose effects can be used to make movies and still frames. The areas involved include architecture, planning and design. Lumion can provide excellent images and combine fast and efficient workflows, saving designers time and effort. The use of lumion to create visual products is also a widely used method in the field of architectural design. Taking into account the important position of BIM technology, lumion provides data exchange plug-ins integrated with Revit to obtain more comprehensive BIM design models and material information. The use of lumion and Revit can quickly replace the material of the converted building model (Dae format), the creation of the terrain, and the layout of the scene. The software's sunlight system can rapidly form high-quality close-to-rendering effects. Live image. It is also possible to perform high-quality rendering of the set point of view and obtain a more detailed rendered image, which of course takes more time. Thanks to its outstanding performance and real-time features, communication between designers and customers is facilitated [5].

Here is another real-time interactive panoramic visualization solution, which is a combination of BIM (Revit) + artlantis studio + Ivisit3D. Artlantis is a visual rendering engine for professional rendering of architectural indoor and outdoor scenes, using FastRadiosity (rapid radiation) engine, extraordinary rendering speed and quality, Artlantis and architectural modeling software such as SketchUp, 3DMAX, ArchiCAD, Revit, etc. With a high level of integration, designers can use BIM software for design and Artlantis for visualization. Artlantis is designed for professional architectural design, providing real-world 3D simulation technology based on hardware and lighting, high-quality animation and still-frame rendering. Based on the 'FastRadio' engine, you can quickly display the rendered effect in the preview window. 3D objects and textures can be applied directly to the preview window with a simple drag and drop. This allows users to quickly set up scenes, control the choice of architectural finishes, and increase rendering quality with superior rendering quality. In addition, Artlantis can also set up any number of cameras and generate $360^{\circ}$ rendered panoramic views of these viewpoint locations to meet the designer or reviewer's detailed view of the architectural design. The flash technology-based panoramic display enables cross-platform applications that can run smoothly on IOS and Android tablet devices, facilitating mobile applications. Sunlight settings based on real geographic location, orientation, and specific date and time can help designers more accurately observe the sunshine and shadow analysis inside and outside the building.

\section{Application Prospect Analysis}

From the perspective of supporting government work, quality supervision agencies apply remote inspection systems for engineering quality, fully apply information technology, innovate and supervise law-enforcement work methods, realize visual supervision of remote video surveillance work, realize electronic filing of engineering data files, and make technical data available. Really reflect the engineering construction process and entities, realize the seamless connection between "build" and "management", effectively alleviate the contradiction between supervision tasks and supervision resource incompatibility, improve the effectiveness of supervision and enforcement, effectively crack down on quality violations and violations, and ensure the realization of quality supervision goals. , Has a good promotion and application prospects and significant economic, social and environmental benefits. From the perspective of the impact on the development of quality supervision industry, through the application and promotion of inspection systems, it can 
solve the key issues of quality supervision in the industry, such as inadequate supervision power, heavy supervision tasks, and form a new type of quality supervision technology system to promote the technical upgrading of quality supervision equipment. The improvement of the overall level of quality supervision has a greater driving effect. From the point of view of supervising law enforcement personnel, the application inspection system can quickly realize the comparison of the project entities and design drawings, and discover that the inspection system can collect the current national effective engineering construction standards and can automatically identify the violation of the project if the site is not constructed according to the design drawings. The requirements of the standard specification, the inspection system can achieve remote visual supervision, supervision of law enforcement personnel can use the inspection system to achieve remote supervision, and reduce a large number of repetitive comparison work, effectively improve the effectiveness of quality supervision and law enforcement.

\section{Conclusions}

BIM technology is destined to become the mainstream of architectural design. This will change the visual production methods and processes of traditional design. As BIM designers need to understand and master a variety of visual production technology methods to meet different needs.

\section{References}

[1] Gao Yonggang, Li Guangjin. Application of BIM Visualization Technology in Hangzhou East Station [J], Civil Engineering and Information Engineering, 2010 (4)

[2] Zhang Yueyan. Based on BIM visualization technology design and application in a commercial center in Fuzhou [J]. Fujian Construction Science and Technology, 2010 (4)

[3] Yu Bingyu, Wang Xiangrong. Analysis of the design flow based on SketchUp software in landscape design [J]. Agricultural Network Information, 2008 (6)

[4] He Yaoguang, Lu Peijun, Hu Yuhua. Based on three-dimensional panoramic visualization platform technology research and practice [J]. Computers and Modernization, 2010 (12)

[5] Zhang Lujing. Based on virtual reality architecture visualization [J]. Shanxi Architecture, 2011 (30) 